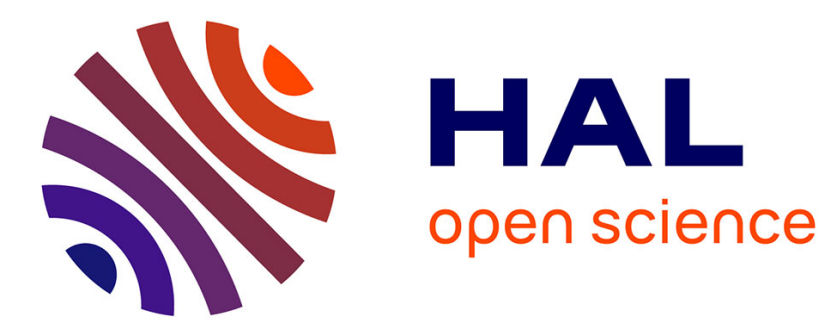

\title{
Le rivage des signes. Remarques sur la ponctuation et l'ailleurs
}

Stéphane Bikialo

\section{To cite this version:}

Stéphane Bikialo. Le rivage des signes. Remarques sur la ponctuation et l'ailleurs. L'information grammaticale, 2004, 102 (1), pp.24-30. 10.3406/igram.2004.2560 . hal-02306376

\section{HAL Id: hal-02306376 \\ https://hal.science/hal-02306376}

Submitted on 5 Oct 2019

HAL is a multi-disciplinary open access archive for the deposit and dissemination of scientific research documents, whether they are published or not. The documents may come from teaching and research institutions in France or abroad, or from public or private research centers.
L'archive ouverte pluridisciplinaire HAL, est destinée au dépôt et à la diffusion de documents scientifiques de niveau recherche, publiés ou non, émanant des établissements d'enseignement et de recherche français ou étrangers, des laboratoires publics ou privés. 


\section{Le rivage des signes. Remarques sur la ponctuation et l'ailleurs} Stéphane Bikialo

\section{Citer ce document / Cite this document :}

Bikialo Stéphane. Le rivage des signes. Remarques sur la ponctuation et l'ailleurs. In: L'Information Grammaticale, N. 102, 2004. pp. 24-30.

doi : 10.3406/igram.2004.2560

http://www.persee.fr/doc/igram_0222-9838_2004_num_102_1_2560

Document généré le 27/09/2015 


\section{LE RIVAGE DES SIGNES}

\section{REMARQUES SUR LA PONCTUATION ET L'AILLEURS}

\section{Stéphane BIKIALO}

Nous avons deux corps : l'un de chair, l'autre de mots; l'un qui s'exprime par le sexe, l'autre par la langue 1.

Les signes de ponctuation apparaissent, au sein de la langue, comme ce qui est au plus près du "corps de chair » 2 . La spécificité de ces signes, à savoir le fait qu'ils ne soient accessibles que par leur signifiant graphique, la trace qu'ils impriment sur la page, la ligne, le mot, implique un rapport à la matérialité, au " corps ", privilégié car plus immédiat 3 , parfaitement évoqué par J. Dürrenmatt dans Bien coupé mal cousu à travers le rapprochement de la réflexion sur la ponctuation au $X \mathrm{X}^{\ominus}$ siècle et de Frankenstein, le corps monstrueux, la créature rapiécée. Ce rapport entre le corps de la langue - mais aussi du texte, de l'œuvre - et la ponctuation est sensible dans les différentes fonctions attribuées à la ponctuation : la ponctuation-respiration (transcription des pauses), la ponctuation-corps conducteur (manière de mimer l'investissement du corps dans ce qu'il a de plus radical), la ponctuation-ossature (signes favorisant la structuration, la hiérarchisation du discours, dans une perspective logique et syntaxique), la ponctuation-vêtement (excroissance, ajout accessoire s'associant au corps du texte sans en devenir partie intégrante, dans une perspective normative), et enfin la ponctuation-greffe ou corps étranger (ajout intégré au corps qui porte atteinte à son intégrité mais lui appartient en propre, ne lui reste pas extérieur) :

la citation est un corps étranger dans mon texte, parce qu'elle ne m'appartient pas en propre, parce que je me l'approprie. Aussi son assimilation, de même que la greffe d'un organe, comportet-elle un risque de rejet contre lequel il faut me prémunir et dont l'évitement est l'occasion d'une jubilation. La greffe prend, l'opération réussit. [...] Le bâti doit disparaître sous la finition, et la cicatrice elle-même (les guillemets) sera un agrément supplémentaire. (Compagnon : 31-32)

La métaphore de la greffe et des guillemets comme cicatrice semble pouvoir être élargie à un bon nombre de signes de ponctuation, en particulier la parenthèse, décrite par

1. B. Noël, Le Tu et le silence, Fata Morgana, $1998: 35$.

2. Dans Le Dictionnaire du diable, A. Bierce propose une origine de la ponctuaton qui rend compte de ce rapport au corps, "l'alimentation des mouches" : "Ces insectes, qui sont toujours attestés dans le voisinage des auteurs, embellissent avec générosité ou parcimonie les manuscrits tout au long de leur composition, et, s'accordant à leurs besoins naturels, mettent en relief avec une sorte d'instinct supérieur l'œuvre des écrivains, à leur insu. " (1911, rééd. Rivages, 1989).

3. "La ponctuation est ce qui règle la langue, mais la règle en mesure; ce qui inscrit en elle notre voix et notre corps " écrivait D. Sallenave dans $L e$ Nouvel Observateur du 18 avril 1991.
S. Pétillon, dans Les Détours de la langue, comme « la greffe d'un dire en plus " (2003: 128) et la note de bas de page, "opération de greffe " selon J. Lefebvre (2004). Considérer les signes de ponctuation comme manifestation d'une greffe revient à les envisager comme les traces graphiques et énonciatives d'une relation entre deux segments dont l'un est posé comme issu de, ou ouvrant sur, un «Ailleurs ». La métaphore spatiale de "l'Ailleurs» - empruntée principalement à $\mathrm{S}$. Pétillon - aspire ainsi à préciser une fonction essentielle de ces signes qui serait de créer un effet d'ailleurs, l'image d'une ouverture du texte sur ce qui ne lui appartiendrait pas en propre, l'atteinte à une sorte d'intégrité fantasmatique du texte, voire du sujet. L'ailleurs pris comme " effet " signifie aussi bien le mouvement d'importation de l'ailleurs dans le corps du texte que le mouvement d'ouverture vers l'ailleurs ${ }^{4}$, d'une part, mais rappelle en outre que la frontière entre le texte et l'ailleurs est instituée par le texte même. Cet effet est lié à la "fonction démarcative" (Anis) de la ponctuation, ainsi qu'à son rôle privilégié dans le signalement de "l'hétérogénéité montrée " (Authier-Revuz), face émergée de "l'hétérogénéité constitutive " dont est affectée toute énonciation, dans la mesure où elle ouvre sur le monde et sur le sujet. Cet ailleurs est donc celui des différents paramètres de la situation d'énonciation (personnes, temps, lieu) ce qui renvoie au caractère souvent déictique des signes de ponctuation, signes d'ostension, de monstration mais aussi de modalisation 5 . Les remarques qui suivent - synthèses d'analyses souvent venues d'ailleurs - ne visent qu'à rendre compte de ce rapport privilégié à l'ailleurs, en insistant sur les différents types et degrés de l'ailleurs mis en œuvre par les signes de ponctuation. On insistera sur le lien étroit entre la nature de la trace graphique (et de l'effort physique qu'elle réclame) et l'opération énonciative à laquelle elle correspond 6 , dans la perspective d'une analyse différentielle des signes, dans l'idée d'une

4. Voir les deux dynamiques énonciatives des signes doubles analysées par S. Pétillon (2003), "l'extraction" (de l'homogène) et "l'insertion " (de l'hétérogène), ainsi que la différence proposée par $\mathrm{S}$. Delesalle entre parenthèses, qui " viennent de l'ailleurs " et tirets, " agent [s] d'extension du texte ", ("Le roman : inclusions et étirements ", dans Authier-Revuz, Lala, 2002 : 173).

5. Cf. les tirets balzaciens destinés à marquer a un mouvement de physionomie " (introduction à la première édition des Chouans en 1829, cité par E. Bordas dans Dürrenmatt (2000)), mais aussi au lien entre les points (interrogatif, exclamatif ou admiratif, suspensif) et les modalités d'énonciation et d'énoncé. 
échelle de soulignement typographique de l'ajout, depuis le niveau zéro de l'absence de tout marquage typographique, en passant par (I) la mise entre virgules, puis par (II) les signes doubles des parenthèses et des tirets, pour en arriver (III) à l'extraction hors de la ligne de la note. (Authier-Revuz, 2002: 161) ${ }^{7}$.

\section{PONCTUATION ET EXTÉRIORITÉ : HÉTÉROGÉNÉITÉ DU DISCOURS ET CEUVRE OUVERTE}

Les signes de ponctuation sont un signal privilégié des « noncoïncidences " (Authier-Revuz) qui parcourent tout dire, qui exhibent et dissimulent - en la circonscrivant en un point du texte - l'hétérogénéité constitutive. Ces non-coïncidences, lieux où l'énonciateur «tient compte de l'autre qui marque son langage ", s'inscrivent dans quatre champs de NON-UN: interlocutive, interdiscursive, entre les mots et les choses, et de la langue à elle-même.

\subsection{Ailleurs interlocutif et interdiscursif}

De quoi est fait un texte? Fragments originaux, assemblages singuliers, références, accidents, réminiscences, emprunts volontaires (Schneider : 12).

L'acte de langage, et l'acte d'écriture en particulier, consiste ainsi à faire sienne cette question : " comment se débrouiller avec du déjà dit ? " (Compagnon, 1979: 12), avec " ce qui parle avant, ailleurs et indépendamment " (M. Pêcheux). Au regard de ce rapport à un ailleurs interlocutif ou interdiscursif (notamment intertextuel) que signalent particulièrement des signes comme les guillemets, les italiques, le soulignement ou les parenthèses, il semble que plus le degré d'ailleurs est important, ou affiché, plus les signes de ponc. tuation sont présents. Ainsi, dans les formes de représentation du "discours autre " 8 , le discours direct peut être démarqué maximalement par l'alinéa et/ou le tiret, et minimalement par la virgule ou le blanc, en passant par les deuxpoints et les parenthèses. La virgule crée une rupture moins forte qui permet selon L. Kaplan d' " être en phase avec le mouvement, avec quelque chose proche du mouvement de pensée. [...] On va avec le personnage " 9 . Ce signalement faible du DD permet donc au discours citant d'atténuer l'effet d'ailleurs, démarche qui culmine dans la «bivocalité » du discours indirect libre, en passant par l'autre forme interprétative, le discours direct libre :

Et moi qui ne puis m'arrêter. Des phrases, des phrases et des phrases. Des phrases jusqu'au vertige. Des phrases jusqu'à l'écœurement [...]. Alors pour m'interrompre, Molinier, moi qui suis un fin connaisseur de Pascal... Et ma répartie immédiate, voulez-vous qu'on croie du bien de vous? N'en dites pas! Et Molinier très pâle, comment osez-vous? Et moi, c'est une citation. De Pascal. Et je pars. (Salvayre : 136)

6. Sur ce lien, voir le bel article de S. Lajus, "La note infrapaginale dans les albums de littérature jeunesse ", La Licorne 67, 2004.

7. S'opposeraient les " signes-claviers " (points, virgule, parenthèses...) aux " signes-barre d'outils " (notes, soulignement, italique, couleurs...), les seconds ouvrant plus fortement sur l'ailleurs.

8. J. Authier-Revuz (2001), dans Une langue : le français, Hachette, p. 192201.

9. Cité dans R. Causse (1998), La langue française fait signe(s), Seuil, p. 175. L. Rosier dans Defays et alii (1998) évoque ce système ancien repris par Leslie Kaplan.
D'une sorte de discours narrativisé (" Des phrases..."), on passe à du DDL (ou du DD très faiblement marqué si l'on considère " pour m'interrompre » et " répartie » comme introducteurs de paroles), qui s'intègre au récit sans guillemets, juxtaposé par une virgule. La parole autre est lissée dans le récit, comme elle l'est dans le discours des personnages avec le jeu sur la citation de Pascal. Inversement, l'emploi fréquent dans la presse d' « îlots textuels " entre guillemets dans du DI apparaît comme un renforcement du caractère autre, au sein d'une démarche d'intégration par la reformulation. D'une forme à l'autre de discours rapporté, il y a une manière de signaler le discours autre comme moins étranger, soit plus proche en ce que les paramètres de la situation d'énonciation ne varient pas tous, soit plus assumé, voire revendiqué comme pouvant être sien. On rappellera la spécificité, dans le cadre du DD, du signe complexe [...] ou [...] qui permet doublement de signaler un ailleurs : ce qui est ainsi posé entre crochets par les points de suspension, c'est ce qui est trop loin du texte pour pouvoir être intégré, c'est la portion de la citation rejetée dans l'ailleurs, qu'il s'agisse de densifier la citation en ne prélevant que ce qui est considéré comme essentiel dans le discours de l'autre, ou encore de censure comme lorsque Lagarde et Michard citent Rabelais :

$\mathrm{Ha}$ ! Badebec, ma mignonne, m'amie, [...] ma tendrette, ma braguette, ma savate, ma pantoufle, 10

Cet exemple témoigne, au niveau de la pratique des éditeurs, du fait que la ponctuation est le lieu privilégié où le lecteur peut investir le texte, que ce soit pour des raisons normatives, comme dans cette évocation d'une lettre dans Ferragus (1833) de Balzac,

Voici textuellement, dans la splendeur de sa phrase naïve, dans son orthographe ignoble, cette lettre, à laquelle il était impossible de rien ajouter, dont il ne fallait rien retrancher, si ce n'est la lettre même, mais qu'il a été nécessaire de ponctuer en la donnant.

ou pour souligner le caractère ouvert de l'œuvre. La ponctuation est l'espace de cette ouverture, en particulier le point de suspension, qui à la fois "présentifie un espace vide (une lacune) [...] où l'attente du sens se trouve différée " et "signale l'infinitude d'un "dire en plus" " 11 , ou les guillemets, la citation étant « un lieu d'accommodation prédisposé dans le texte", qui "donne rendez-vous» (Compagnon, 1979 : 23). Le signe de ponctuation qu'est le plus chargé de "remplir " le lecteur est sans doute le blanc comme le montrent, diversement, les pages blanches proposées ou envisagées par Sterne et Furetière dans leurs romans ou le blanc des journaux brésiliens censurés 12. Dans La Belle Hortense, Roubaud joue avec humour de cet espace en créant la notion d'entre-deux-chapitres:

10. Le texte intégral est: “ Ha, Badebec, ma mignonne, ma mie, mon petit con (toutefois elle en avait bien trois arpents et deux sexterées), ma tendrette..." ".

11. M.-C. Lala, "L'ajout entre forme et figure... ", dans Authier-Revuz, Lala (2002 : 185).

12. Voir E. Orlandi (1996), Les Formes du silence, éditions des Cendres, p. 94 . 
Les auteurs de romans [...] ont rarement l'obligeance, nous dirons même la courtoisie, de ménager ainsi, comme nous, des espaces de repos à leurs lecteurs [...]. Ces espaces verts du roman, innovation dont nous proposons le modèle à nos contemporains, collègues et successeurs, nous suggérons de leur donner le nom d'“ entre-deux-chapitres ". Chacun y est invité; on peut s'y reposer, méditer sur les bancs de quelques questions, avant de reprendre sa marche le long du récit. (Roubaud, 1985 : 73).

Ce néologisme ponctuant se présente comme une explicitation et une mise en scène (ironique?) de l'œuvre ouverte, "projet d'un message doté d'un large éventail de possibilités interprétatives ", comprenant les « œuvres "ouvertes" en mouvement " qui se caractérisent par " une invitation à faire l'œuvre avec l'auteur » (Eco, $1965: 11,35)$.

\subsection{Le réel et le sujet comme ailleurs}

Les signes de ponctuation ont partie liée avec d'autres formes d'hétérogénéité : celles du rapport du sujet de l'écriture au réel, à la langue et à lui-même. Face à l'écart irréductible entre l'ordre de la langue et du réel, rester au plus près du réel implique ainsi d'essayer de mettre en scène cette noncoïncidence (Bikialo, 2003), ce qui passe notamment par la ponctuation chez Simon :

plus tard, [...] il se rendit compte qu'il avait fabriqué au lieu de l'informe, de l'invertébré, une relation d'événements telle qu'un esprit normal (c'est-à-dire celui de quelqu'un qui a dormi dans un lit, s'est levé, lavé, habillé, nourri) pouvait la constituer après coup, à froid, conformément à un usage établi de sons et de signes convenus, [...] tandis qu'à la vérité cela n'avait ni formes définies, ni noms, ni adjectifs, ni sujets, ni compléments, ni ponctuation (en tout cas pas de points), ni exacte temporalité, ni sens, ni consistance sinon celle, visqueuse, trouble, molle, indécise, de ce qui lui parvenait à travers cette cloche de verre (Simon, 1989 : 286-287)

L'absence ou la raréfaction de la ponctuation forte est ainsi perçue (et employée dans l'écriture) comme une manière d'être au plus près de ce qui n'est pas verbal, considéré comme Ailleurs du texte (et de la langue). "Mon cher ami, j'ai ponctué, parce que somme toute il ne faut pas nous mettre tout le monde à dos 13 ", écrivait Mallarmé à Dujardin en 1886. Posons que se « mettre tout le monde à dos ", c'est aussi s'interdire de pouvoir écrire quelque chose du monde; d'où le "en tout cas pas de points" de Simon : la virgule (importante chez cet auteur), précisément par sa polysémie, et la hiérarchisation hésitante qu'elle pose, permet de rester au plus près du réel :

La surponctuation apparaît comme le point ultime de la dislocation de la fiction d'un langage capable de littéralité et donc d'une littérature qui dirait le monde connaissable. Avec moi le chaos... (Dürrenmatt, $1998: 89$ ).

Cet ailleurs est aussi celui du sujet qui se pense avec distance, comme autre, fonction que remplit souvent la parenthèse, comme espace de réflexivité, ce dont témoigne l'extrait précédent de L'Acacia, à travers le décrochement, l'énoncé méta-énonciatif introduit par «c'est-à-dire ", ou encore l'écri-

13. Cité par J. Scherer (1977), Grammaire de Mallarmé, Nizet, p. 203. ture de $M$. Foucault où « les éléments décrochés donnent à voir les cheminements, les trajets d'une écriture qui "raisonne" et dans laquelle le "je" s'efface pour laisser place à l'analyse " (Pétillon, 2003 : 6). Dans le même esprit, la marge est l'espace de commentaire du sujet comme l'ont montré Herschberg Pierrot (1985) et Neefs (1989). Ce que révèlent ces parenthèses et ces notes marginales, c'est la présence de l'Autre en soi, le clivage foncier du sujet

" Je est un autre. " Pas vrai : « je est d'autres ". D'autres choses, d'autres odeurs, d'autres sons, d'autres personnes, d'autres lieux, d'autres temps. [...] Comment peut-on être toujours conséquent pendant six cent vingt-trois pages. Voilà ce que je me demande, moi qui ne suis jamais le même pendant dix minutes à la file, moi qui ne suis pas le même pendant la durée d'un millième de seconde, puisque je ne suis pas moi. (Simon, 1947 : 174)

Ce clivage du sujet trouve son expression graphique radicale dans Le Corps lesbien de M. Wittig et les barres obliques / parfois appelées, de manière parfaitement adaptée en l'occurrence, slash (briser en anglais) :

$\mathrm{J} / \mathrm{e} \mathrm{m} /$ /interroge dans le silence dans le manque de trace, $\mathrm{j} / \mathrm{inter}$ roge une absence si étrange qu'elle m/e cause un trou au-dedans de m/on corps. (Wittig, $1973: 31$ )

Selon Drillon, la barre oblique peut être un "interrupteur " employé pour relier deux termes opposés et exclusifs ("dénotation/connotation "), ou «indique un choix" entre deux termes proches, ou encore rapproche deux mots différents. Aucune de ces valeurs discursives n'est ici pertinente, où la barre renvoie au clivage du sujet (on pense à la barre de fraction, qui divise), transformant un morphème en phonèmes, une unité distinctive porteuse de sens en deux non porteuses de sens dans le cas de "je " ou la marque de l'élision (l'apostrophe) en marque de clivage : c'est la même barre oblique que l'on trouve dans $S / Z(1970)$ qui signifie un enjeu d'identité :

$S$ et $Z$ sont dans un rapport d'inversion graphique : c'est la même lettre vue de l'autre côté du miroir : Sarrasine contemple en Zambinella sa propre castration. Aussi la barre (/) qui oppose le $S$ de SarraSine et le $Z$ de Zambinella a-t-elle une fonction panique : c'est la barre de censure, la surface spéculaire, le mur de l'hallucination, le tranchant de l'antithèse, l'abstraction de la limite, l'oblicité du signifiant, l'index du paradigme, donc du sens. (CEuvres complètes III : 207).

Dans Le Corps lesbien, la barre a bien cette " fonction panique " liée au rapport à l'autre, à l'ailleurs : c'est un corps pénétré, disloqué, voire mutilé qui est mis en scène, un corps qui cherche à se situer par rapport à un «tu " - non barré lui et non nommé - auquel le texte est adressé. Ce «j/e » apparaît ainsi comme signifiant un rapport de soi à soi, de clivage, de démembrement interne, mais aussi de soi à l'autre, et de l'autre en soi. C'est autour de cette question de l'identité que tourne le texte, la barre oblique témoignant parfaitement, et avec le caractère implacable de la trace graphique nette qu'elle crée, de cette tension entre le Même et l'Autre (le Même de l'homosexualité et l'Autre du corps de la femme aimée ou de son propre corps considéré avec distance) :

$\mathrm{j} / \mathrm{e}$ fais éclater les petites unités de m/on m/oi, j/e suis menacée, $\mathrm{j} / \mathrm{e}$ suis désirée par toi $[. ..] \mathrm{j} / \mathrm{e}$ suis introduite par toi, $\mathrm{j} / \mathrm{e}$ dois à 
présent lutter contre l'éclatement pour continuer m/a perception globale, j/e te rassemble dans tous $\mathrm{m} / \mathrm{es}$ organes, $\mathrm{j} / \mathrm{e} \mathrm{m} / \mathrm{e}$ clate, j/e m/e rassemble. (op. cit. : 109)

On sait par ailleurs, grâce à l'édition proposée par F. Bon, que ce signe/est celui employé par Rabelais dans les énumérations :

J'ai (répondit Gargantua) par longue \& curieuse expérience inventé un moyen de me torcher le cul, le plus royal/le plus seigneurial/le plus excellent, le plus expédient, qu'on ait jamais vu ${ }^{14}$.

La barre oblique relie des termes à la fois équivalents d'un point de vue syntaxique et référentiel, qui fait relever cette configuration de la nomination multiple, configuration qui signifie parfaitement ce rapport du Même et de l'Autre, qui semble être la valeur de base de ce signe de ponctuation. En outre, dans ce dernier cas, il permet une prolifération syntagmatique qui relève de l'ajout, de la catalyse.

\section{PONCTUATION ET CATALYSE.}

Pour Barthes, la phrase et le récit sont infiniment catalysables, peuvent être pourvus d'une infinité d'expansions :

Certaines [fonctions] constituent de véritables charnières du récit (ou d'un fragment de récit); d'autres ne font que « remplir » l'espace narratif qui sépare les fonctions-charnières : appelons les premières des fonctions cardinales (ou noyaux) et les secondes, eu égard à leur nature complétive, des catalyses. [...]; les catalyses disposent des zones de sécurité, des repos, des luxes; ces "luxes " ne sont cependant pas inutiles [...] : une notation, en apparence explétive, a toujours une fonction discursive [...] : le noté apparaissant toujours comme du notable, la catalyse réveille sans cesse la tension sémantique du discours, dit sans cesse : il y a eu, il va y avoir du sens; la fonction courante de la catalyse est donc, en tout état de cause, une fonction phatique (pour reprendre le mot de Jakobson) : elle maintient le contact entre le narrateur et le narrataire. [...] on le sait, c'est ce qui se passe pour la phrase, faite de propositions simples, compliquées à l'infini de duplications, de remplissages, d'enrobements, etc. : comme la phrase, le récit est infiniment catalysable 15.

La ponctuation paraît être un vecteur essentiel de cette catalyse : du point qui note à la fois l'arrêt et le nouveau départ à la parenthèse et sa récursivité, en passant par la virgule qui permet des énumérations théoriquement infinies. Cette vertu catalytique est liée aux œuvres ouvertes qui, «bien que matériellement achevées, restent ouvertes à une continuelle germination de relations internes" (Eco: 35$)$ et touche de nouveau au corps comme le souligne cette formulation (érotique) de la "catalyse voluptueuse " dans Sade, Fourier, Loyola (1971):

La saturation de toute l'étendue du corps est le principe de l'érotique sadienne : on essaye d'employer (d'occuper) tous ses lieux distincts. Ce problème est celui-là même de la phrase [...] : la phrase (littéraire, écrite) est elle aussi un corps qu'il faut catalyser, en remplissant tous ses lieux premiers (sujet-verbe-complément) d'expansions, d'incises, de subordonnées, de détermi-

14. Rabelais (1534), Gargantua, rééd. par F. Bon, POL, 1992, cité par L. Bianchi (2002: 27).

15. Barthes (1966), "Introduction à l'analyse structurale des récits", CEurres complètes II, p. 840-843. nants; certes, cette saturation est utopique, car rien ne permet (structuralement) de terminer une phrase (CEuvres complètes III: 814).

La valeur de déclencheur catalytique de la ponctuation est particulièrement sensible dans les manuscrits (marge, becquets...) et dans les notes et les liens hypertextuels (soulignement) permettant un enchâssement d'espace autres.

\subsection{Les manuscrits, espace catalytique}

Les manuscrits mettent en scène plusieurs degrés de l'ailleurs. Dans la page, le pavé d'écriture est souvent mis en relation avec le reste de l'espace paginal par des signes comme la rature (qui ouvre sur l'espace interlinéaire) ou les becquets, les flèches, qui ouvrent sur l'espace marginal, "vaste réservoir d'idées" et

espace dialogique entre discours et métadiscours [...], entre sujet biographique et narrateur, entre situation d'énonciation [...] et énoncé (voir chez Stendhal : " 3 juillet, fatigué après 26 pages") (Grésillon, $1994: 60$ ).

La marge est donc une marque de ponctuation blanche dont la fonction est d'accueillir un ailleurs du texte - souvent susceptible de s'intégrer au texte ou de n'être un ailleurs que graphique et non syntaxique comme Herschberg Pierrot (1985) l'a montré au sujet des notes marginales du manuscrit de Bouvard et Pécuchet - que cet ailleurs soit celui d'un autre sujet (glose marginale allographe des manuscrits médiévaux) ou celui du sujet dialoguant avec lui-même, entre deux éditions (Montaigne et Stendhal sur l'exemplaire Chaper de La Chartreuse de Parme) ou entre deux états de son manuscrit :

La marge est comme la place laissée pour le destinataire [...]. Un dialogue passe, par dessus la frontière qui borde le texte et sa marge, par dessus la frontière du temps. [...] La marge est, sur la page, la place où peuvent cohabiter une différence et un dialogue, $[. .$.$] elle est le pli d'un texte vers un autre. (Neefs,$ $1989: 61)$

C'est pourquoi on peut distinguer les auteurs qui prévoient une place à l'ailleurs en réservant une marge importante (Stendhal, Flaubert, Hugo, Simon) et ceux qui saturent la page (Sartre, Comte, Walser) comme pour interdire cette irruption de l'ailleurs. Si la marge est un signe de ponctuation en soi, sa situation induit aussi la présence d'autres signes, spécifiques à l'écriture manuscrite, comme le becquet, "signe métascriptural qui symbolise le lieu d'insertion d'un ajout ou la nouvelle place assignée à une unité différée. " (Grésillon, 1994 : 241), ou la flèche (analysée par F. Boch dans Defays et al., 1998). Ces signes ont pour fonction de relier le texte et son ailleurs, la nature graphique de cet accompagnement, qui va du texte à l'ailleurs ou de l'ailleurs au texte, étant fondamentale dans l'analyse du degré du décrochement énonciatif : les manuscrits donnent ainsi à voir un ailleurs flottant que sont les listes paradigmatiques, dans une errance de mots souvent non reliés au texte, et un

16. Cf. aussi le "plan de montage " de La Route des Flandres de C. Simon qui attribue une couleur à chaque personnage. 
ailleurs intégré ou en instance d'intégration à la fois graphique et syntaxique. Si le texte imprimé nivelle ou atténue ces degrés d'ailleurs, l'avancée des travaux en génétique textuelle et des techniques éditoriales, cumulée avec la pratique de certains auteurs, que ce soient les volumes-manuscrits de Stendhal ou la revendication d'un " nouveau genre littéraire " chez Ponge (Le Pré, Comment une figue de parole, etc.) permettent la parution de textes où peut être intégrée une nouvelle pratique ponctuante. Ainsi de La Table de Ponge présenté par J. Thibaudeau, rééditée en 2002 dans une collection relativement accessible ("Nrf ", Gallimard) et qui utilise certains signes issus des manuscrits inhabituels dans les imprimés (flèches, accolades pour les ajouts interlinéaires). Plus fondamentalement encore, les éditions Al Dante viennent de rééditer Nouvelles Impressions d'Afrique de Roussel (mise en couleur et postface de J. Sivan) en respectant le vœu essentiel, jusque là non satisfait, de l'auteur, à savoir l'alternance de couleurs. Ce poème en quatre chants, célèbre pour sa "forêt concentrique de parenthèses" (M. Foucault), est un modèle, dans sa genèse même, de l'ouverture vers l'ailleurs à travers les variations de la ponctuation. Issu d'une double contingence éditoriale (le texte était jugé trop court et Roussel l'augmenta donc non seulement de texte mais d'illustrations), le texte (au niveau de la phrase comme de l'œuvre) se gonfle, selon un principe thématisé dans le poème et sensible dans les tirets, les deuxpoints, les notes de bas de page et les parenthèses/couleurs :

Et le petit chapeau - desquels nous extrayons

Quel que soit notre bord d'intimidants rayons -

(extraire à tout propos est naturel à l'homme;

II extrait : de ce rien, la chute d'une pomme,

Une loi qui le voue à l'immortalité;

D'une fable ou d'un conte une moralité;

Du grêle épouvantail, simple croix qui se dresse

- Sa tenue accusant la plus noire détresse -

((Que d'aspects prend la croix! un groupement astral (Roussel, $1963: 21)$

Les décrochements énonciatifs marqués par les parenthèses permettent un creusement progressif de la linéarité et un enchâssement de voix, à partir d'un mot (" extraire ", " croix") dans un mouvement de généralisation et de commentaire méta. Ce "topogramme lié " (Anis, 1989) que représente la couleur, qui se greffe sur le mot, semble particulièrement apte à noter la multiplication des sujets ou des voix 16 . Dans Tokyo infra-ordinaire, l'utilisation des couleurs - entre autres principes - permet à J. Roubaud de hiérarchiser son texte par degrés successifs de commentaires méta-énonciatifs sur le texte en train de s'écrire :

47 station 2 Shin Ôkubo (mon point de départ généralement, en fait, après 1996)

Je commence un poème de métro:

471 qu'est-ce qu'un poème de métro?

472 définition de la forme, inventée par Jacques Jouet, de r'Oulipo, selon la forme

4721 cest un poème de métro:

473

"Un poème de métro est un poème commencé dans le métro, pendant le temps d'un voyage.
Un poème de métro compte autant de vers que votre voyage compte de stations moins un.

Le premier vers est composé dans votre tête entre les deux premières stations de votre voyage (en comptant la station de départ).

Il est transcrit sur le papier quand la rame s'arrête à la station deux.

Le deuxième vers est composé dans votre tête entre les stations deux et trois de votre voyage.

II est transcrit sur le papier quand la rame s'arrête à la station trois. Et ainsi de suite.

II ne faut pas transcrire quand la rame est en marche. II ne faut pas composer quand la rame est arrêtée.

Le dernier vers du poème est transcrit sur le quai de votre dernière station. [...]

48 (first line, Shin Ôkubo)

Dans ce wagon il n'y a que des places debout

49 station 3 Takadanobaba [...]

c'est un wagon d'un type spcial; je lui attribuerai la formule $7+5$

491 j'ai défini antérieurement, dans des notes non reproduites ici, un système d'identification des wagons de métro ou de train

492 «spcial » n'est pas une erreur de frappe; je respecte la notation originelle, celle du moment de la composition du poème de métro

4921 ce que ne fait pas JJ (Jacques Jouet, de I'Oulipo).

17

Dans ce texte $\mathrm{sp}(\mathrm{a}) \mathrm{tial}$, la structuration par la numérotation, les alinéas et les couleurs renvoie non seulement au genre et au thème du texte (un poème de métro) mais aux différentes strates énonciatives. Kyrielle (Nous, 2003) de Roubaud reprend ce principe puisqu'il s'agit d'une œuvre polyphonique, destinée explicitement à un opéra à cinq voix. On retrouve là un rapport à l'oralité qui n'est plus de l'ordre de la ponctuation-respiration mais d'une performance à venir, comme dans les poèmes sonores, "partitions" (titre d'un ouvrage de B. Heidsieck) toujours susceptibles de variations:

Les mot, pour tant, c'est pas c'qui manque!

mais : quand faut les trouver (

:( ) les chercher () les trouées !... (Bobillot:29)

Comme l'écrit J.-P. Bobillot au sujet de ce poème, “ la version proposée ici, que l'on peut selon toute vraisemblance considérer comme définitive a été réalisée courant 1997 ", la modalisation épistémique "selon toute vraisemblance » soulignant ce caractère par essence inachevé et infini de l'œuvre.

\subsection{Ailleurs et hypertexte}

L'exemple le plus abouti de cette ouverture sur l'ailleurs est sans doute actuellement "l'hyperlivre» de R. Camus, Vaisseaux brûlés, texte évolutif en expansion permanente et infinie - les " 999 » paragraphes le soulignant - installé sur Internet en 1998 :

«1. Ne lisez pas ce livre! Ne lisez pas ce livre!" Tel était le premier des 999 paragraphes de $P A$ (Petite Annonce), volume publié aux éditions POL en 1997. Depuis lors P.A. s'est vu installé sur le net, l'espace qui lui était le plus approprié, et même le seul adéquat, peut-être, tant le cours du récit et du sens, en cette

17. Le premier niveau (47) est en noir, le second (47.1. et 47.2.) en rouge, le troisième (47.2.1.) en bleu, le quatrième (49.2.1.1.) en vert. 
Annonce, loin d'être tendu de façon linéaire, du début vers une fin, comme dans les livres ordinaires, était fertile en carrefours, au contraire, en chemins de traverse, en pertes, en cavatines, en cavernes, en abymes - parenthèses dans la parenthèse et notes à la note à la note à la note, indéfiniment. En ce transfert d'un monde à l'autre, PA est devenu Vaisseaux brûlés, gigantesque atelier en expansion permanente, où chacun des paragraphes de l'ouvrage initial, chacune de ses phrases, chacun de ses mots, a vocation à engendrer une littérature arborescente, au gré de remords et d'ajouts. (Camus, $2000: 4$ ème de couverture)

Ces « remords et ajouts " sont adjoints au texte selon le principe du lien hypertextuel, marqué graphiquement par le soulignement (conservé dans la publication papier, ce qui témoigne de sa valeur de signal, d'effet d'ailleurs) :

Le soulignement a l'effet d'un embrayeur, d'une marque de l'énonciation dans l'énoncé par laquelle l'auteur donne à entendre à quelque lecteur quelque chose en plus de la signification et qui lui est irréductible... (Compagnon : 20)

Cet « en plus de la signification " est constitutivement, dans le cas du soulignement hypertextuel, celui d'un ailleurs, que cet ailleurs renvoie à des lieux autres (comme le site des éditions POL, l'association culturelle « Le pli » dont s'occupe l'auteur ou encore sa propre adresse, manière singulière de formuler le "pacte autobiographique " et rappel que PA est aussi fait de petites annonces, d'ordre sexuel et professionnel, dans un jeu de l'offre et de la demande qui renvoie à l'ouverture de l'œuvre vers le lecteur) ou à d'autres lieux du texte :

454. Si je parle mal en public [...] c'est à cause, encore une fois, d'une simultanéité fatale, entre les tentations d'expression, parmi les exigences (ou qui m'apparaissent telles dans ces moments affreux) de ce qu'il conviendrait d'exprimer. Chaque mot est un carrefour en étoile. Chaque parole est trop dire, ou pas exactement, pas assez. Chaque idée appelle son histoire en incises, le récit de sa gestation, ses contradictions dépassées, les réserves qu'elle suscite encore. C'est un scrupule qui me fait bégayer, puis un remords de ce scrupule, puis le regret de ce remords (178).

457. J'exagère, évidemment, car je ne bégaie pas, pas encore. [...] Si je viens de parler de bégaiement, c'est par emportement rhétorique. II arrive - c'en est un exemple - que la phrase tourne toute seule, se cherche des harmoniques, des symétries, des points de chute possibles, pour retomber sur ses pattes. Ce faisant elle s'éloigne de la vérité, bien entendu; en général, au moins, mais pas toujours : $[\ldots](219,243)$. [...]

460. La meilleure traduction littéraire ou formelle de la métaphore des chemins de traverse (ou bien ce seraient les chemins de traverse, au contraire, qui seraient le référent d'on ne sait quelle métaphore...), ce serait les parenthèses, bien sûr, les incises, les crochets, les parenthèses dans les crochets, parenthèses dans les parenthèses, l'abyme, l'anacoluthe mais surtout la note, et la note à la note, et la note à la note à la note.

L'évocation de différents degrés de l'ailleurs s'accompagne de sa représentation dans l'écriture (segments juxtaposés par des virgules, parenthèses et tirets doubles), le soulignement marquant le degré le plus fort. Ce signe qui peut porter sur un mot, un chiffre, un syntagme ou une phrase, possède la caractéristique d'être «à la fois lié et détaché
[...] solidaire du segment alphagraphique mais matériellement distinct " (Anis, $1989: 37$ ), statut qu'on reliera à son tracé, qui demande à la fois un certain effort graphique notamment lorsqu'il porte sur un long segment - et se situe aisément dans la continuité du geste d'écriture, par sa nature horizontale, linéaire. A ce titre, il est à la fois un signal fort de " décrochement » et en lien matériel étroit avec l'élément qu'il ouvre. On pourrait formuler la valeur de base de ce signe par le terme de "déviation", chemin secondaire et provisoire (par rapport au chemin normal) mais aussi nécessaire, ne pouvant pas ne pas être emprunté. Par cette valeur, on retrouve les deux emplois majeurs du soulignement : la mise en valeur - qu'on retrouve chez Camus sur « mais pas toujours " - et le lien hypertextuel - pour les renvois de paragraphe ou la phrase "chaque mot est un carrefour en étoile". Le lien hypertextuel est à cet égard très proche de « l'appelrenvoi de note " 18 mis au jour par J. Lefebvre, signe relevant « à la fois de la dimension de «l'espace visuel » du livre et de l'ordre de la langue " :

Signe bifrons - l'appel de note regardant vers le texte, et le renvoi de note vers la note - l'appel-renvoi de note a pour particularité d'articuler une note à un texte c'est-à-dire de permettre la greffe, en un point précis du corps du texte - et ce grâce à l'appel de note - de ce qui est montré comme un « autre du texte ", la note. (Lefebvre, 2004 : 39)

Le soulignement hypertextuel renvoie toutefois à un degré d'ailleurs plus fort que la note de bas de page, mais proche de la note placée en fin de chapitre ou de volume. Comme le lien hypertextuel qui permet un parcours du texte non linéaire (tout en indiquant, par la couleur, les chemins déjà empruntés), la note peut imposer un mode de lecture spécifique, en particulier dans des textes où les notes prennent le dessus sur le texte central - Feu pâle de V. Nabokov, La Maison des feuilles de M.-Z. Danielewski) - voire se substituent au texte, renvoyant à un texte absent (L'Interdit de G. Wajcman, "Banlieue » de Paul Fournel, texte sans centre - comme son titre l'indique - qui se présente comme une série de variations sur tout ce qui est à la périphérie du texte, avec au centre sept pages blanches, avec notes). L'ailleurs devient le centre, le texte.

\section{"Si loin, si proche".}

Le Farghestan avait dressé devant moi des brisants de rêve, l'au-delà fabuleux d'une mer interdite; il était maintenant une franche accore de côte rocheuse, à deux journées de mer d'Orsenna. (J. Gracq, Le Rivage des Syrtes, Corti, 1951 :199)

L'Ailleurs, inaccessible, lointain se révèle proche, juste séparé par un peu de mer (de blanc) et une côte accore (du noir). Ce rapport variable à l'ailleurs, entre distance et proximité pourrait être une métaphore de la ponctuation. $\mathrm{Ce}$ « rivage des signes ", on a pu en donner quelques exemples : de la virgule, des points, de la barre oblique, etc. qui indiquent une bifurcation, une rupture dans la linéarité à la note, à l'espace marginal, au soulignement (hypertextuel) qui permet-

18. Pour le lien entre ces signes, voir Dürrenmatt (2004) qui évoque, chez Camus, la note comme espace de "l'ouverture à tous les textes possibles" (59) et l'article d'O. Ertzscheid dans le même recueil. 
tent d'opérer une véritable sortie de la linéarité, en passant par les parenthèses, les tirets doubles et les crochets qui instaurent un "décrochement " qui " creuse " la linéarité (Pétillon), la ponctuation possède bien cette dimension d'ouvrir, par degrés, vers un ailleurs (un autre texte, une autre énonciation). "Mots sans mots", " histoires sans paroles" (N. Catach), les signes de ponctuation renvoient au motus et bouche cousue, au silence comme tentation absolue face à l'omniprésence écrasante de l'interdiscours, des mots des autres, de l'écart entre la langue et le réel. Mais face au silence, ces signes accordent et imposent, dans leur matérialité, une place à cet ailleurs.

\section{Stéphane BIKIALO Paris III/SYLED/FORELL}

\section{RÉFÉRENCES BIBLIOGRAPHIQUES}

Anis J., 1989. « De certains marqueurs graphiques dans un modèle linguistique de l'écrit ", DRLAV $41: 33-52$.

Authier-Revuz J., 1995. Ces mots qui ne vont pas de soi. Boucles réflexives et non-coincidences du dire, Larousse.

Authier-Revuz J., Lala M.-C. dir., 2002, Figures d'ajout. Phrase, texte, écriture, Presses de la Sorbonne Nouvelle.

Barthes R., 2002. CEuvres complètes, Seuil.

Bianchi L., 2002. «Ponctuation ip (ou) r, q-uo-i! », R de Rée/ volume $P: 20-30$.

Bikialo S., 2003. Plusieurs mots pour une chose. De la nomination multiple au style de Claude Simon Thèse de doctorat. Poitiers.

Bobillot J.-P., 2000. Crevez le matelas de mots \& autres poèmes, Atelier de l'Agneau.
Defays J.-M., Rosier L., Tilkin F. dir., 1998. A qui appartient la ponctuation?, Duculot.

Camus R., 1998... Vaisseaux brûlés, http://perso.wanadoo.fr/ renaud.camus.

Camus R., 2000. Ne lisez pas ce livre!, POL.

Compagnon A., 1979. La Seconde main ou le travail de la citation, Seuil.

Drillon J., 1991. Traité de la ponctuation française, Gallimard.

Dürrenmatt J., 1998. Bien coupé mal cousu, PU Vincennes.

Dürrenmatt J. dir., 2000. La Ponctuation, La Licorne 52, Poitiers.

Dürrenmatt J., 2004. "La note comme lieu d'autoréférence ", La Licorne 67 : L'Espace de la note, PU Rennes : 51-64.

Eco U., 1965. L'CEuvre ouverte, Seuil, "Points".

Grésillon A., 1994. Éléments de critique génétique, PUF.

Herschberg Pierrot A., 1985. "La marge des notes ", Leçons d'écriture. Ce que disent les manuscrits, dir. Grésillon, Werner, Minard.

Lefebvre J., 2004. " " Note " et « note " : proposition de défrichage linguistique ", La Licorne 67 : 27-50.

Neefs J., 1989. "Marges", De la lettre au livre. Sémiotique des manuscrits littéraires, éditions du CNRS : 57-88.

Pétillon-Boucheron S., 2003. Les Détours de la langue. Étude sur la parenthèse et le tiret double, Peeters, "BIG».

Roubaud J., 1985. La Belle Hortense, Ramsay, rééd. Seghers, 1990, "Points".

Roubaud J., 2003. Tokyo infra-ordinaire, Inventaire/Invention éditions.

Roussel R., 1932. Nouvelles Impressions d'Afrique, rééd. J.J. Pauvert, 1963 et Al Dante, 2004.

Salvayre L., 1995. La Puissance des mouches, Seuil, "Points". Schneider M., 1985. Voleurs de mots, Gallimard.

Simon C., 1947. La Corde raide, Le sagittaire.

Simon C., 1989. L'Acacia, Minuit.

Wittig M., 1973. Le Corps lesbien, Minuit. 